\title{
Monitoring the effectiveness of HIV and STI prevention initiatives in England, Wales, and Northern Ireland: where are we now?
}

\author{
A E Brown, S E Tomkins, L E Logan, D S LaMontagne, H L Munro, V D Hope, A Righarts, \\ J E Blackham, B D Rice, T R Chadborn, P A Tookey, J V Parry, V Delpech, O N Gill, K A Fenton
}

Sex Transm Infect 2006;82:4-10. doi: 10.1136/sti.2005.016386

Primary and secondary prevention are essential components of the response to HIV and sexually transmitted infections (STIs). We present findings from nationally implemented HIV/STI prevention interventions. In 2003, of those attending STI clinics at least $64 \%$ of men who have sex with men (MSM) and $55 \%$ of heterosexuals accepted a confidential HIV test; $88 \%$ of all HIV infections in women giving birth in England were diagnosed before delivery; $85 \%$ of MSM eligible for hepatitis B vaccination received a first dose of vaccine at their first STI clinic attendance; $74 \%$ of STI clinic attendees for emergency appointments, and $20 \%$ of those for routine appointments were seen within 48 hours of initiating an appointment; the National Chlamydia Screening Programme in England found a positivity of $10 \%$ and $13 \%$ among young asymptomatic women and men, respectively. Prevention initiatives have seen recent successes in limiting further HIV/STI transmission. However, more work is required if current levels of transmission are to be reduced.

See end of article for authors' affiliations

Correspondence to: Alison E Brown, HIV and STI Department, Centre for Infections, 61 Colindale Avenue, London NW9 5EQ, UK; alison.brown@ hpa.org.uk

Accepted for publication 26 September 2005
$\mathrm{P}$ revention is an essential component of the response to HIV and sexually transmitted infection (STI) transmission. Despite the availability of effective antiretroviral therapy (ARV), HIV infection is only treatable provided that an assiduous routine of medication is followed indefinitely (often with adverse side effects $\left.^{1}\right)$. HIV care and treatment are expensive ${ }^{2}$ and ARV resistance is thought to be increasing in England. ${ }^{3}$ STI treatment costs are also substantial $^{4}$ and if left untreated, can have serious long term sequelae ${ }^{5}$ and possibly facilitate the transmission of others, including HIV.

For HIV/STIs, primary prevention targets uninfected individuals, for instance, by reducing risk factors for disease acquisition. Examples include diagnosing HIV in pregnant women (to prevent vertical transmission), hepatitis B vaccination, and harm reduction measures (for example, needle exchanges). Secondary prevention targets infected individuals, aiming to reduce onward disease transmission or re-infection. Examples include the promotion of sexual health screeningfor example, chlamydia screening among young people and promoting voluntary confidential HIV testing (VCT) in STI clinics. An HIV diagnosis provides access to ARV, a timely STI diagnosis usually leads to treatment, and both allow an opportunity for partner notification and behaviour change counselling.

Major challenges remain in ensuring that prevention initiatives are effective. They need to be accessible (particularly for higher risk populations who may be socially vulnerable); timely; comprehensive (address all modes of HIV/STI transmission); implemented through functioning health systems; and subject to monitoring and evaluation.

The Health Protection Agency and its collaborators monitor the effectiveness of some primary and secondary prevention efforts in addition to providing national HIV/STI surveillance data. We present findings from nationally coordinated prevention monitoring programmes. This paper does not present an overview of all HIV/STI prevention activities that occur in the United Kingdom, but summarises information on prevention monitoring and disease outcomes, to demonstrate recent progress, and highlight areas that need further work.

\section{DATA SOURCES}

In the United Kingdom, the majority of HIV/STI prevention initiatives are implemented through STI clinics, primary care, and other community based services. The Health Protection Agency and its collaborators use nationally coordinated information systems to monitor prevention initiatives. Prevention monitoring systems ${ }^{7}$ (summarised in table 1) and their objectives ${ }^{2} 8$ are generally separate from the variety of infection surveillance systems used, but can overlap.

\section{HIV testing}

Monitoring the uptake of VCT and antenatal HIV screening relies on data from the Unlinked Anonymous Prevalence Monitoring Programme (UAPMP) surveys. ${ }^{9}$

The UAPMP survey of STI clinic attendees measures HIV prevalence (including undiagnosed

Abbreviations: ARV, antiretroviral therapy; IDUs, injecting drug users; MSM, men who have sex with men; NCSP, National Chlamydia Screening Programme; NSHPC, National Study of HIV in Pregnancy and Childhood; SOPHID, Survey of Prevalent HIV Infections Diagnosed; STI, sexually transmitted infections; UAPMP, Unlinked Anonymous Prevalence Monitoring Programme; VCT, voluntary confidential HIV testing 


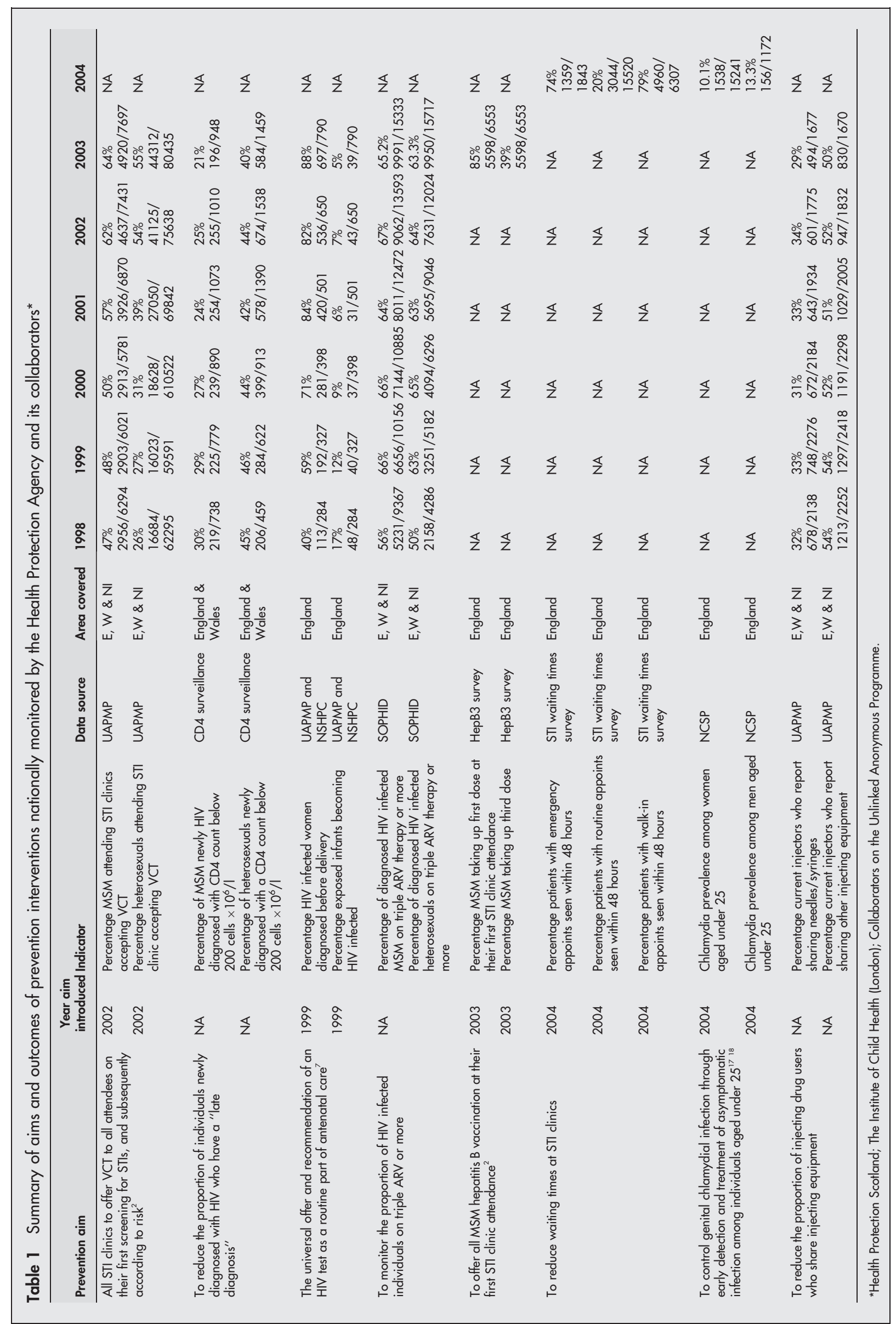


HIV infection) in attendees of 16/232 STI clinics in England, Wales, and Northern Ireland undergoing syphilis tests. ${ }^{10}$ Residual blood from syphilis testing is irreversibly unlinked from patient identifiers and anonymously HIV tested. Retained information includes sexual orientation and sexual health screen uptake (including VCT, further details are available on the STI website).

The UAPMP surveys of pregnant women (utilising residual serum from newborn infant dried blood spots, covering $80 \%$ of births in England and Scotland) provide a proxy measure of HIV prevalence in the overall population. Live births to diagnosed HIV infected women in the United Kingdom are reported to the National Study of HIV in Pregnancy and Childhood (NSHPC). ${ }^{8}$ The proportion of HIV infected women diagnosed before delivery is calculated by aligning NSHPC reports ${ }^{11}{ }^{12}$ with the total number of births to diagnosed and undiagnosed HIV infected women. The number of infants who become infected themselves is estimated by applying UK specific observed transmission rates for infants born to diagnosed and undiagnosed HIV infected women. ${ }^{13}$

\section{CD4 surveillance}

CD4 T lymphocyte counts in HIV infected individuals (CD4 Surveillance Scheme) are reported from 60 laboratories in England and Wales (representing approximately two thirds of all reported new diagnoses) and are used to monitor trends in immunosuppression at HIV diagnosis. ${ }^{14}$ Individuals who have a CD4 count below 200 cells $\times 10^{6} / 1$ (the recommended threshold for beginning therapy ${ }^{15}$ ) at HIV diagnosis are categorised as having a "late HIV diagnosis."

\section{Antiretroviral therapy monitoring among diagnosed HIV infected individuals}

The annual Survey of Prevalent HIV Infections Diagnosed (SOPHID) provides a census of the total number of individuals receiving HIV related care in England, Wales and Northern Ireland. ${ }^{16}{ }^{17}$ Subsidiary information is collected on ARV uptake and most recent CD4 count.

\section{Uptake of hepatitis B vaccination among men who have sex with men}

The HepB3 survey monitors hepatitis B vaccination uptake among eligible men who have sex with men (MSM) on their first STI clinic attendance. Since the study started in 2003, 187/209 English clinics have participated.

\section{Chlamydia screening programme}

The National Chlamydia Screening Programme (NCSP) aims to control genital chlamydial infection through early detection of asymptomatic infection ${ }^{18} 19$ outside STI clinic settings. From April 2003 to March 2004, 302 screening venues (including contraceptive clinics, GPs, young people's services, and termination clinics) participated from 10 programme areas. The target population is sexually active individuals aged under 25. Demographic and behavioural data are also collected.

\section{STI clinic waiting times}

Since 2004, a biannual audit of waiting times is conducted among all new attendees at all STI clinics in England for 1 week. Age and sex specific waiting times are collected for each clinic as discreet categorical units.

\section{Behavioural and serosurveillance of injecting drug users}

The UAPMP survey of injecting drug users (IDUs) collects self reported behavioural data (for example, injecting equipment sharing) in addition to measuring the prevalence of blood borne viruses among injectors attending 63 specialist services in England, Wales, and Northern Ireland. ${ }^{20}$ Sharing rates are calculated for those who reported injecting in the previous 4 weeks.
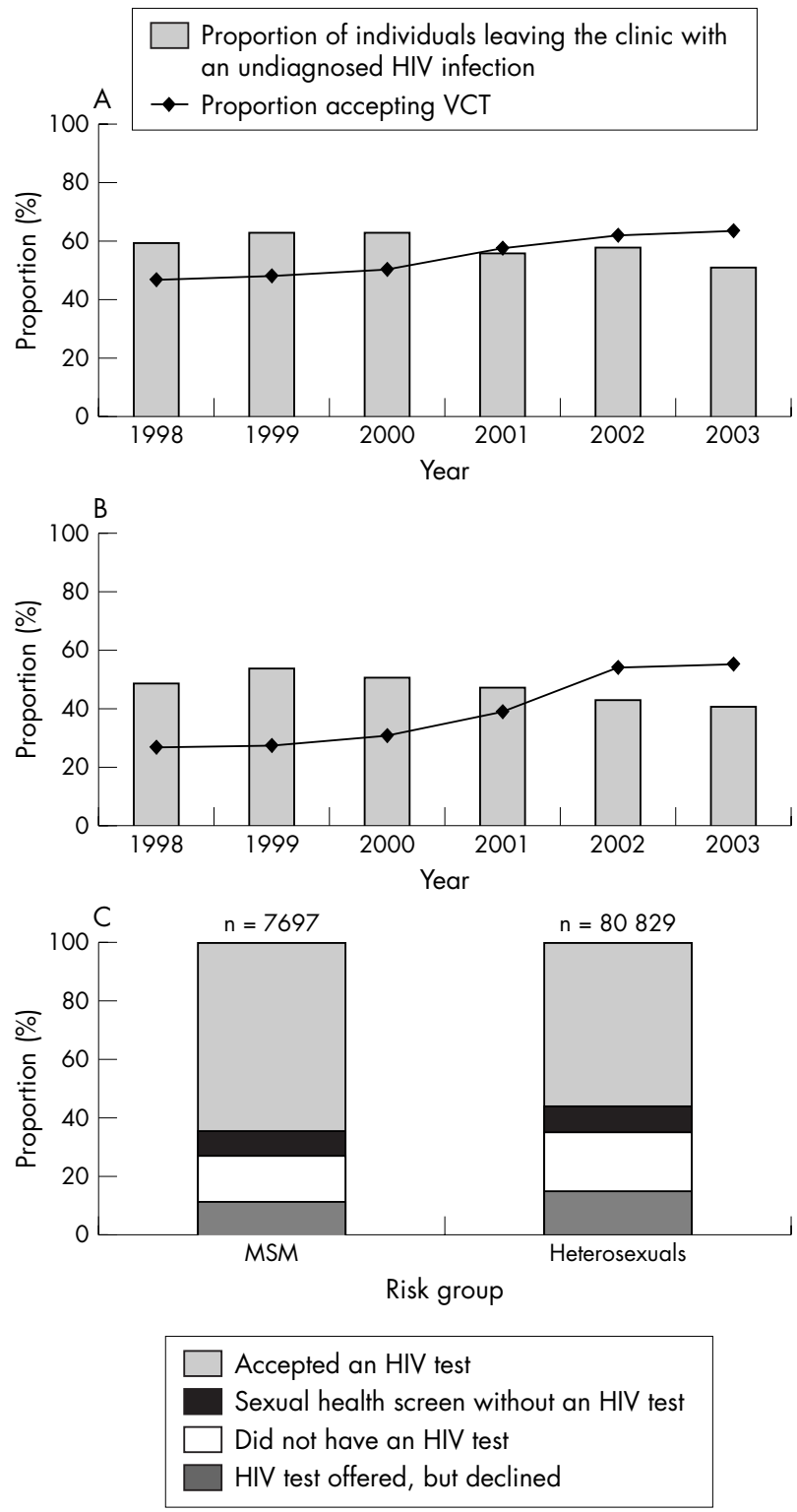

Figure 1 Proportion* of STI clinic attendees, accepting VCT†, and proportion of HIV infected individuals who leave the clinic with their HIV infection remaining undiagnosed, England, Wales, and Northern Ireland 1998-2003. (A) MSM, (B) heterosexuals, (C) proportion* of STI clinic attendees accepting VCT $\dagger$ by exposure category, 2003. ( ${ }^{*}$ Excludes HIV infected individuals who were previously diagnosed. Patients for whom conflicting information was provided on HIV testing were excluded. †Attending 16 GUM clinics in England, Wales, and Northern Ireland.) Data source: Unlinked anonymous programme.

Descriptive epidemiology is the focus of the paper, but $95 \%$ confidence limits (95\% CI) have been used to supplement main findings from the NCSP and the sentinel Unlinked Anonymous STI and IDU survey. All other prevention monitoring systems are comprehensive.

\section{RESULTS: PREVENTION MONITORING UPDATE VCT uptake}

Overall, VCT uptake rose by $17 \%$ (95\% CI 15\% to 19\%) from $47 \%(2956 / 6294)$ in 1998 to at least 64\% (4920/7697) in 2003 among MSM and by $28 \%$ (95\% CI $27 \%$ to $28 \%$ ) from $27 \%$ ( $16886 / 62295)$ in 1998 to at least 55\% (44 312/80 435) among heterosexuals (fig $1 \mathrm{~A}$ and $\mathrm{B}$ ). Of those who did not have VCT, at least 29\% (817/2777) of MSM and 31\% (11 312/ 


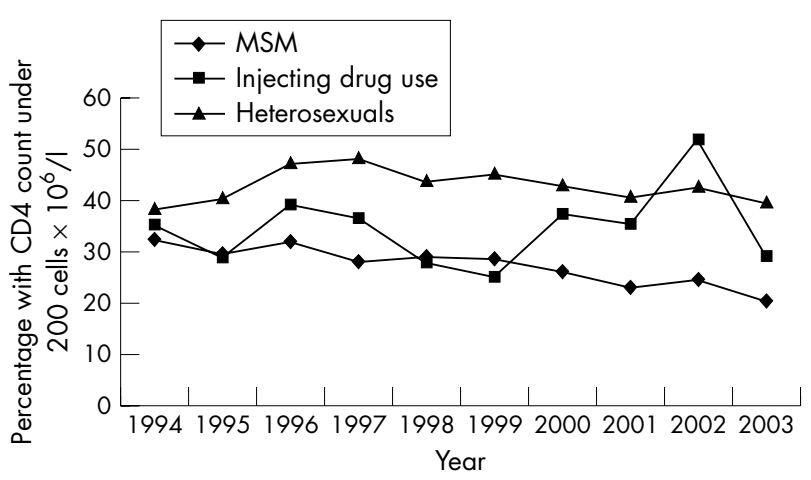

Figure 2 Late diagnosis* of HIV infection by exposure category, England and Wales, 1994-2003. (*Percentage of patients with CD4 count under 200 cells $\times 10^{6} /$ I within 90 days of diagnosis.) Data source: CD4 surveillance scheme.

36 123) of heterosexuals are known to have been offered, but declined, VCT (fig IC). Of those that declined VCT, 7\% (56/ $817)$ of MSM and $1 \%(83 / 11312)$ of heterosexuals were HIV infected.

The proportion of HIV infected individuals who could have been diagnosed during their attendance, but who left the clinic remaining unaware of their HIV infection, fell by $9 \%$ (95\% CI 1\% to $17 \%$ ) from $60 \%$ (165/276) in 1998 to $51 \%$ (161/ 317 ) in 2003 among MSM and may have fallen by 7\% (95\% CI $-1 \%$ to $15 \%)$ from $48 \%(104 / 217)$ in 1998 to $41 \%$ (160/ 394) in 2003 among heterosexuals.

\section{Late diagnoses}

In 2003, 33\% (995/2982) of people with newly diagnosed HIV infection in England and Wales had CD4 counts below 200 cells $\times 10^{6} /$ l. MSM are increasingly being tested at earlier

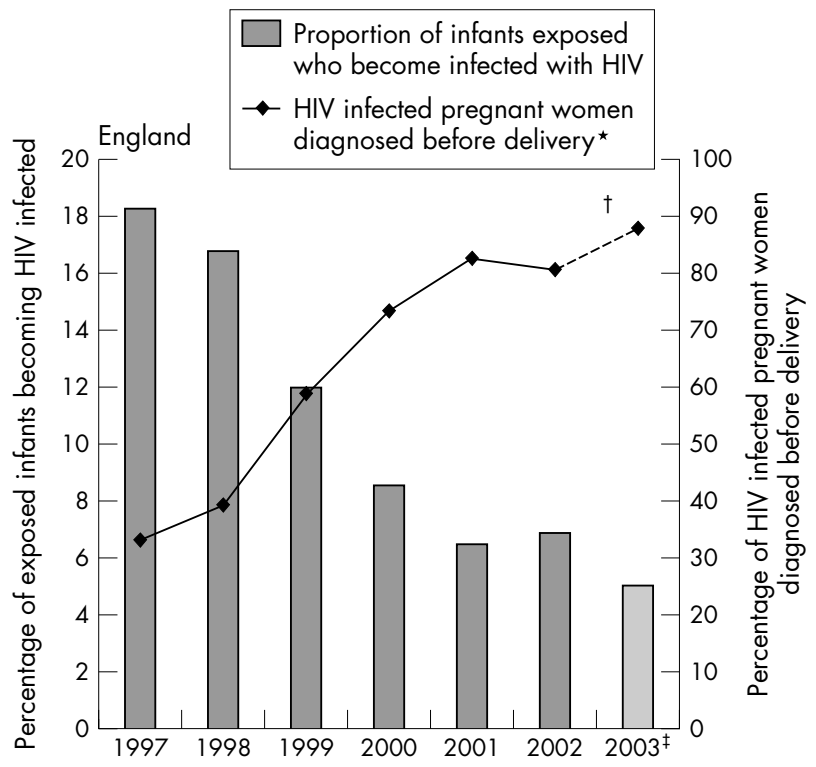

Figure 3 Estimated proportion of HIV infected women diagnosed before delivery ${ }^{*}$, and of exposed children becoming HIV infectedt, England, 1998-2003. ('Includes previously diagnosed and those diagnosed through antenatal testing. †Assumes a vertical transmission rate of $26.5 \%$ in undiagnosed women and $2.2 \%$ in diagnosed women. ${ }^{13}$ ¥These data contain reports received by the end of September 2004 and are subject to reporting delay, particularly for 2003.) Data source: Unlinked Anonymous Programme and the National Study of HIV in Pregnancy and Childhood. stages of their infection ${ }^{21}$ (fig 2). HIV infected heterosexuals were more likely to be diagnosed late.

\section{Antenatal HIV testing}

In England, 88\% (697/790) of HIV infected women who gave birth in 2003 are calculated to have had their infection diagnosed before delivery. The majority of births to HIV infected women occurred in London where 89\% (455/511) of women were diagnosed before delivery (fig 3).

The proportion of children exposed to maternal HIV infection who acquire HIV is decreasing. Based on current estimated detection rates, $5 \%$ of children exposed to vertical transmission would have been infected in England in 2003, compared to $17 \%$ in 1998.

\section{Uptake of antiretroviral therapy}

Of MSM receiving HIV care, 65\% (9991) were receiving at least three antiretroviral drugs in 2003 compared to 56\% (5231) in 1998. Among heterosexuals, equivalent figures were $63 \%$ (9956) and 50\% (2158). Thirty three per cent of MSM (5065) and 35\% (5466) of heterosexuals were not receiving HIV therapy in 2003.

\section{Uptake of hepatitis B vaccine among MSM}

MSM were considered to be eligible for hepatitis B vaccination (dose 1) if they were not known to be either immune or fully/partially vaccinated. Overall, 85\% (5598/6553) of eligible MSM were vaccinated with dose 1 .

MSM eligible for the third vaccine dose (dose 3) included those who had had fewer than three doses, but excluded those who had had a booster dose, and those known to have immunity through blood testing following previous doses. The coverage rate for dose three was 39\% (2588/6624) overall but showed regional variation (fig 4$)$. Nearly one half $(46 \%$, 2588/5669) of MSM eligible for dose 1 completed the three dose course.

\section{STI clinic waiting times}

Nationally, $74 \%$ (1359/1843) of emergency appointments, $79 \%$ (4960/6307) of people attending walk-in clinics, and 20\% (3044/15 520) of people with routine appointments were seen within 48 hours. Lower proportions of 16-24 year olds and women of any age were seen within 48 hours. Full results have been published elsewhere. ${ }^{22}$

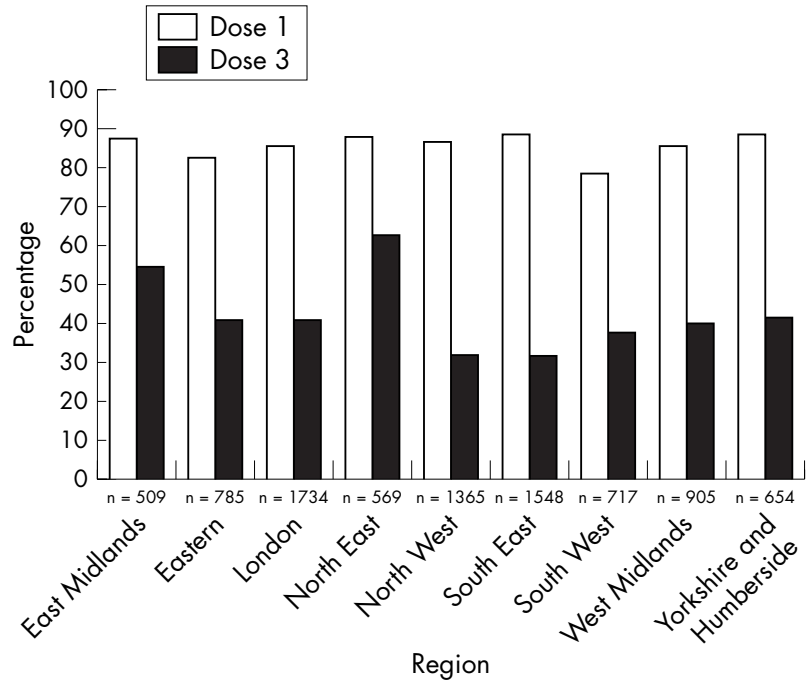

Figure 4 Coverage rates for hepatitis B vaccination (first and third dose) among eligible MSM attending STI clinics for the first time, by region, England, 2003. Data source: HepB3 survey. 


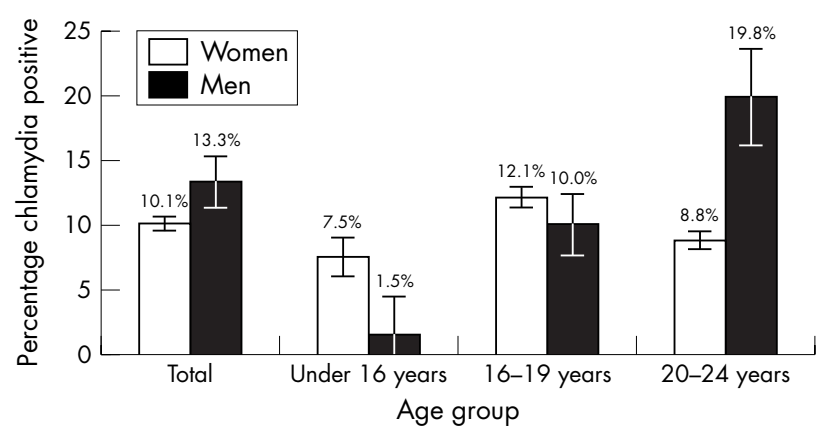

Figure 5 Chlamydia positivity, opportunistic screening outside STI clinics by sex and age group, England, April 2003-March 2004. Data source: National Chlamydia Screening Programme.

\section{Chlamydia screening programme}

In England, 16413 young people were screened for chlamydia outside STI clinic settings during April 2003-March $2004 .{ }^{19}$ A $10 \%$ (1538/15 241, 95\% CI 9.6\% to $10.6 \%$ ) and $13 \%$ (156/1 172, 95\% CI $11.4 \%$ to $15.4 \%$ ) positivity among women and men aged under 25 was found respectively (fig 5). Women aged 16-19 were more likely to test positive for chlamydia than those aged 20-24; men aged 20-24 were twice as likely to be infected as younger men.

\section{Risk behaviours among IDUs}

Thirty per cent $(494 / 1677,95 \%$ CI $28 \%$ to $32 \%$ ) of injectors reported sharing needles and syringes in 2003; a level similar to that observed since $1998 .{ }^{23}$ In $2003,85 \%$ (331/384) of IDUs who had first injected in the previous 3 years reported ever having accessed a needle exchange service.

\section{DISCUSSION}

Monitoring individual HIV/STI prevention initiatives in 2003 demonstrated successes. VCT uptake among STI clinic attendees was at the highest level ever recorded. Almost $90 \%$ of HIV infected women had their infection diagnosed before delivery in England leading to an increase in the proportion able to take advantage of interventions to prevent vertical transmission (for example, ARV and avoiding breast feeding), thereby decreasing the proportion of infants who become infected. The proportion of diagnosed HIV infected individuals on at least triple therapy has increased from around half in 1998 to almost two thirds in 2003. HIV infected MSM are being diagnosed at an earlier stage of their infection. Targeted screening for chlamydia has identified many asymptomatic infections that otherwise may have been missed.

However, high proportions of STI clinic attendees were not seen within 48 hours of seeking an appointment, delaying access to treatment and potentially heightening the risk of onward transmission. ${ }^{24}$ The elevated levels of injecting equipment sharing among IDUs is concerning.

\section{Are prevention initiatives reducing transmission?}

Despite the individual success of many prevention initiatives, the number of HIV/STI diagnoses are increasing annually. In 2003 there were 6606 new HIV diagnoses, more than double the 2835 diagnoses in $1998 .^{7}$ From 2002 to 2003, diagnoses of chlamydia at STI clinics rose by $8 \%$, and syphilis by $28 \%$ among men and 32\% among women.

The number of diagnoses is determined by interactions between transmission dynamics, migration of individuals from high prevalence areas, and the relative effectiveness of targeted testing. Increases in diagnoses represent both successes in increased testing in high risk populations as well as continued disease transmission within that population. The relative contribution of these factors is difficult to disentangle.

However, HIV incidence may be increasing among MSM attending STI clinics, ${ }^{25}$ whilst HIV prevalence among recently initiated injectors (those who began injecting in the previous 3 years) was at the highest level recorded in 2003, perhaps a result of elevated levels of equipment sharing. ${ }^{20}$

The reasons for continuing transmission despite apparently successful prevention initiatives require consideration:

\section{Do prevention monitoring systems show the whole} picture?

In the United Kingdom, methods for monitoring prevention initiatives are pragmatic, simple, inexpensive and reach accessible populations-not necessarily those at greatest risk. Consequently, data are generally derived from those using health services, and will exclude high risk populations who have poor access to services.

Monitoring systems also collect limited data. While rates of STIs are higher among black and ethnic minority populations, $^{26}{ }^{27}$ few UK prevention monitoring systems collect ethnicity data, making it difficult to monitor the specific impact of prevention initiatives on such vulnerable populations.

Each prevention monitoring system has its own limitations.

\section{HIV testing}

All patients included in the Unlinked Anonymous STI survey are undergoing syphilis tests, therefore, VCT in this population may not represent all STI clinic attendees. The survey cannot monitor the frequency of repeat HIV testing (which may positively bias the results), or outcomes among first time attendees.

Diagnosis detection rates among HIV infected pregnant women are calculated by aligning diagnosis reports to the NSHPC with UAPMP prevalence data. Since data are anonymised, records are not individually matched. Limited mismatching may occur with respect to time and geography. Detection rates are minimum estimates and may rise as late reports are received by the NSHPC.

CD4 surveillance suggests MSM with HIV are being diagnosed earlier in their infection. However, the high proportion of heterosexuals categorised as having a "late diagnoses" may not accurately reflect recent efforts in VCT promotion; a high proportion of heterosexuals are infected abroad and may not have been resident in the United Kingdom long enough to have had an earlier diagnosis.

\section{ARV}

HIV infected individuals accessing health care show an increase in ARV uptake. However, it is difficult to calculate what proportion of HIV infected individuals should be on therapy. Guidelines state that individuals who have a CD4 count below 200 cells $\times 10^{6} / 1$ should begin therapy. ${ }^{15}$ Such guidelines are not directed from a public health perspective to prevent HIV transmission, but on the basis of individual need/readiness; patients may delay, interrupt, or stop therapy for many reasons.

The proportion of diagnosed HIV infected individuals with low CD4 counts on ARV is not routinely calculated because these specific fields are incomplete for a minority of records. In the future, this proportion may be calculated through cross linking to other HIV reporting databases. ${ }^{28}$

\section{Hepatitis $B$ vaccination}

The HepB3 study demonstrates that high proportions of MSM are vaccinated with dose 1 on their first STI clinic attendance, but lower proportions complete the three dose course. Patient identifiers are not collected, so it is impossible 
to monitor movement of patients between clinics. This, combined with reporting delay, may lead to an underestimate of the true performance.

\section{STI clinic waiting times}

Although the national waiting times survey show a high proportion of attendees cannot get a timely appointment, it is not possible to calculate the median waiting time since data is collected in categories.

\section{Chlamydia screening programme}

While the NCSP has improved access for chlamydia screening since its implementation in England, it is not currently possible to calculate national coverage. When the programme is fully rolled out throughout England, coverage will be calculated by dividing the number of people aged 16-24 screened by the total eligible population (sexually active population aged 16-24).

\section{Risk behaviour monitoring}

The Unlinked Anonymous IDU survey only includes those in contact with services for drug users and therefore the data may not be generalisable to all injectors, specifically, those not in contact with services, who may have different levels of risk behaviour

\section{Are prevention initiatives effective?}

The effectiveness of prevention initiatives to reduce transmission requires assessment. For instance, although the promotion of VCT has reduced the proportion of HIV infected individuals leaving the clinic remaining undiagnosed, it may not target those who have been recently infected, who may be more infectious. Research is required to elucidate the role of "recently HIV infected" people in contributing onward HIV transmission. For IDUs, there is evidence of a recent shift in needle exchange provision towards pharmacy based services. ${ }^{29}$ Studies suggest that IDUs using pharmacy based services may be more likely to share equipment owing to absence of harm reduction counselling in these settings. ${ }^{30}$

\section{Are prevention initiatives implemented on the correct scale?}

Since the prevalence of an infection drives onward transmission, recent increases in the prevalent pool of diagnosed and undiagnosed infections may limit the ability of prevention programmes to reduce transmission levels.

For example, the success in reducing the proportion of infants exposed to maternal HIV infection who become infected, has not substantially reduced the absolute number, because prevalence among pregnant women has increased. Continuing investment in prevention activities is essential, and activities need to adapt to meet the challenge of the evolving epidemics.

\section{What next?}

While individual prevention initiatives have had an impact, and rates of ongoing transmission would have been higher in their absence, investment needs to be strengthened and sustained in order to reduce HIV/STI transmission. Current prevention initiatives partially accommodate the diversity of populations at high risk of infection, but require flexibility if they are to match the evolving epidemic. Evaluation of prevention initiatives, both individually and in combination, is needed to measure how much they reduce transmission. Novel monitoring tools require development to assess prevention initiatives aimed at populations who have poor access to health services, but who play an important part in HIV/STI transmission.

Local and national surveillance systems are essential in ensuring that the effectiveness of prevention initiatives are continually reviewed and updated to meet the diversity of needs of the populations at risk of HIV/STIs.

\section{ACKNOWLEDGEMENTS}

We thank David Goldberg, Daniel Thomas, Brian Smyth, Sarah Dougan, Elizabeth Rudd, Christine McGarrigle, and all others who contributed to the writing and editing of the annual report Focus on Prevention.

We gratefully acknowledge the continuing collaboration of the Sexually Transmitted and Blood-Borne Viruses Laboratory, Centre for Infections, Health Protection Agency and of clinicians, microbiologists, immunologists, public health practitioners, midwives, and other colleagues who contribute to the surveillance of HIV/STIs in the United Kingdom. We are also grateful to the English Department of Health for funding specific surveys.

We would like to thank our collaborating centres for HIV and AIDS surveillance in the UK: Health Protection, Scotland; The Institute of Child Health (London); The UK Haemophilia Centres Doctors Organisation; members of the Scottish ISD(D) 5 Collaborative Group; Collaborators on the Unlinked Anonymous Programme (a full list of collaborators available at: www.hpa.org.uk/infections/ topics_az/hiv_and_sti/hiv/epidemiology/ua.htm).

Confidential reports of HIV infected pregnant women are collated at the Institute of Child Health by the National Study of HIV in Pregnancy and Childhood through surveillance schemes run in collaboration with the Royal College of Obstetricians and Gynaecologists and the British Paediatric Surveillance Unit of the Royal College of Paediatrics and Child Health. Research at the Institute of Child Health benefits from R\&D funding received from the NHS Executive.

Finally, we thank Dr Helen Ward and Susie Huntington for their useful comments on drafts of this paper.

\section{CONTRIBUTORS}

$\mathrm{AB}, \mathrm{LL}, \mathrm{SL}, \mathrm{HM}, \mathrm{VH}, \mathrm{AR}, \mathrm{BR}$, and $\mathrm{TC}$ analysed the data from the Unlinked Anonymous STI clinic survey, Unlinked Anonymous pregnant women survey, National Chlamydia Screening Programme, HepB3 survey, Unlinked Anonymous IDU survey, STI waiting times survey, SOPHID and CD4 surveillance scheme respectively with support from VD, NG, and KF; PT coordinates the National Study of HIV in Pregnancy and Childhood and collaborated with the analysis of the Unlinked Anonymous Pregnant Women Surveys; VD oversees the data for SOPHID, CD4 surveillance, and the NCSP; NG is the programme manager and is responsible for data from the Unlinked Anonymous Programme; JP is responsible for laboratory aspects for the Unlinked Anonymous Surveys and assisted with the interpretation of data; all authors were involved in interpretation of the results and drafting the paper with ST and JB substantially contributing; $\mathrm{AB}$ undertook the main writing of the paper.

\section{Key messages}

- Prevention activities have seen recent successes in limiting further HIV/STI transmission in England, Wales, and Northern Ireland

- High proportions of STI clinic attendees are having voluntary confidential HIV tests. HIV infected pregnant women are diagnosed before delivery; MSM are receiving hepatitis $B$ vaccinations at their first clinic attendance

- Prevention monitoring activities may be missing at-risk populations who are likely to have an important role in onward HIV/STI transmission

- Existing prevention initiatives may only be having a limited effect on the current rate of HIV/STI transmission and require development to match evolving epidemics 
An information page is on the STI website, www.stijournal.com/supplemental

\section{Authors' affiliations}

A E Brown, S E Tomkins, L E Logan, D S LaMontagne, H L Munro, V D Hope, A Righarts, J E Blackham, B D Rice, T R Chadborn, V Delpech, O N Gill, K A Fenton, HIV and STI Department, Health Protection Agency Centre for Infections, 61 Colindale Avenue, London NW9 5EQ, UK

V D Hope, Centre for Research on Drugs and Health Behaviour, Imperial College London, UK

P A Tookey, Institute of Child Health (ICH), University College London, UK

J V Parry, The Sexually Transmitted and Blood-borne Viruses Laboratory, Health Protection Agency Centre for Infections, UK K A Fenton, The Centre for Sexual Health and HIV Research, Department of Primary Care and Population Sciences, Royal Free and University College Medical School, London, UK

\section{REFERENCES}

1 Carosi G, Torti C. "Solving the puzzle" of current HAART: what key strategic trials are needed? New Microbiol 2004;27:127-30.

2 Department of Health. The national strategy for sexual health and HIV. London: Department of Health, 2001.

3 Scott P, Anrold E, Evans B, et al. Surveillance of HIV antiretroviral drug resistence in treated individuals in England: 1998-2000. J Antimicrob Chemother 2004;53:469-73.

4 Office of Health Economics. Cost of treatment for sexually transmitted diseases. London: Office of Health Economics, 1995

5 Cates W, Wasserheit JN. Genital chlamydial infections: epidemiology and reproductive sequalae. Am J Obstet Gynaecol 1991;164:1771-81.

6 Nusbaum MR, Wallace RR, Slatt LM, et al. Sexually transmitted infections and increased risk of co-infection with human immunodeficiency virus. J Am Osteopath Assoc 2004; 104:527-35.

7 The UK Collaborative Group for HIV and STI surveillance. Focus on prevention. HIV and other sexually transmitted infections in the United Kingdom in 2003. London: Health Protection Agency Centre for Infections, November, 2004

8 Ades $\mathrm{AE}$, Davidson CF, Holland FJ, et al. Vertically transmitted HIV infection in the British Isles. BMJ 1993;206:1296.

9 Nicoll A, Gill ON, Peckham CS, et al. The public health applications of the unlinked anonymous seroprevalence monitoring programme for HIV in the United Kingdom. Int J Epidemiol 2000;29:1-10.

10 Catchpole MA, McGarrigle CA, Rogers PA, et al. Serosurveillance of prevalence of HIV infection in homosexual men with acute sexually transmitted infection. BMJ 2001;321:1318-20.

11 Ades AE, Davidson CF, Holland FJ, et al. Vertically transmitted HIV infection in the British Isles. BMJ 1993;206:1296.
12 Cliffe S, Tookey PA, Nicoll A. Antenatal detection of HIV: national surveillance and unlinked anonymous survey. BMJ 2001;323:376-7.

13 Duong T, Ades A, Gibb DM, et al. Vertical transmission rates for HIV in the British Isles: estimates based on surveillance data. BMJ 1999:319:1227-9.

14 Gupta SB, Dingley SD, Lamgni TL, et al. The national CD4 surveillance scheme for England and Wales. Commun Dis Public Health 2001;4:27-32.

15 Pozniak A, Gazzard B, Anderson J, et al. British HIV Association (BHIVA) guidelines for the treatment of HIV-infected adults with antiretroviral therapy. HIV Med 2003;4(Suppl 1):1-41.

16 McHenry A, Macdonald N, Sinka K, et al. National assessment of prevalent diagnosed HIV infections. Commun Dis Public Health 2000;3:277-81.

17 Rice BD, Payne $\amalg$, Sinka K, et al. The changing epidemiology of prevalent diagnosed HIV infections in England, Wales and Northern Ireland. Sex Transm Infect 2005;81:223-9.

18 Department of Health. National chlamydia screening programme in Englandprogramme overview, core requirements and data collection. 2nd ed. London: DoH, 2004.

19 LaMontagne DS, Fenton KA, Randall S, et al. Establishing the national chlamydia screening programme in England: results from the first full year of screening. Sex Transm Infect 2004:80:335-41.

20 Health Protection Agency, Scottish Centre for Infection and Environmental Health, National Public Health Service for Wales, Communicable Disease Surveillance Centre Northern Ireland, Centre for Research on Drugs and Health Behaviour, Unlinked Anonymous Steering Group. Shooting up; infections among injecting drug users in the United Kingdom 2003. London: Health Protection Agency, October, 2004

21 Chadborn TR, Baster K, Delpech VC, et al. No time to wait: how many HIVinfected homosexual men are diagnosed late and consequently die? (England and Wales 1993-2002). AIDS, 2005 25, 19:513-20.

22 www.hpa.org.uk/infections/topics_az/hiv_and_sti/epidemiology/ wtimes.htm.

23 Hope VD, Rogers PA, Jordan LF, et al. Sustained increase in the sharing of needles and syringes among drug users in England and Wales. AIDS 2002; 16:2494-6.

24 White PJ, Ward H, Cassell JA, et al. Vicious and virtuous circles in the dynamics of infectious disease and the provision of health care; gonorrhea in Britain as an example. J Infect Dis 2005; 192:824-36.

25 Murphy G, Charlett A, Brown AE, et al. Is HIV incidence increasing in MSM attending GUM clinics in England, Wales and Northern Ireland? Commun Dis Public Health 2004;7:11-14.

26 Brown AE, Sadler KE, Tomkins SE, et al. Recent trends in HIV and other STIs in the United Kingdom: data to the end of 2002. Sex Transm Infect 2004;80:159-66

27 Low N, Sterne JA, Barlow D. Inequalities in rates of gonorrhoea and chlamydia between black ethnic groups in south east London: cross sectional study. Sex Transm Infect 2001;14:63-9.

28 Patel B, Chadborn TR, Rice BD, et al. Epidemiological and clinical trends in levels of antiretroviral therapy uptake, using national surveillance data (19982002). BHIVA conference 2004 15-17 April;10-18.

29 McVeigh J, Beynon C, Bellis MA. New challenges for agency based syringe exchange based syringe based exchange schemes analysis of 11 years of data (1991-2001) in Merseyside and Cheshire, United Kingdom. Int J Drug Policy 2003; 14:399-405.

30 Thein HH, Denoe M, van Beek I, et al. Injecting behaviour of injecting drug users at needle and syringe programmes and pharmacies in Australia. Int J Drug Policy 2003;14:399-405. 